\title{
STUDY ON MAIZE LEAF MORPHOLOGICAL MODELING AND MESH SIMPLIFICATION OF SURFACE
}

\author{
Xinyu Guo ${ }^{1, *}$, Chunjiang Zhao ${ }^{1}$, Boxiang Xiao, ${ }^{1,2}$ Shenglian $\mathrm{Lu}^{1}$, \\ Changfeng $\mathrm{Li}^{1}$ \\ ${ }^{1}$ National Research Center for Information Technology in Agriculture, Beijing, China, 100097 \\ ${ }^{2}$ Institute of computer technology, Dalian University of Technology, Dalian, China, 116024 \\ * Corresponding author, Address: P.O. Box 2449-26, Beijing, 100097, P. R. China, Tel: +86- \\ 10-51503422, Fax: +86-10-51503750,Email: guoxy@nercita.org.cn
}

Abstract: According to the need of canopy visualization calculation in the digital plant research, we introduced a method, using Non-Uniform Rational B-Splines (NURBS) interpolation and multi-line segment splitting algorithm, to reconstruct the $3 \mathrm{D}$ morphological structure of maize leaf with a complexity controllable mesh. Using the data cloud obtained by digitizer, construct the surface of maize leaf by calculating the knot vectors and reverse calculating surface control points by difference calculation. The final visualization effect is realistic. According to leaf morphological characteristics, leaf surface mesh can be simplified by using inverse calculation of multi-line segment splitting algorithm, and the surface main characteristics can be maintained simultaneously. This method can be used in canopy visualization calculation and light distribution calculation. Results showed that it can improve the calculation efficiency obviously without increase the calculation error.

Keywords: maize leaf; NURBS; geometric modeling; mesh simplification; light distribution calculation

\section{INTRODUCTION}

The morphological structure of plant is always the important subject in biology, agronomy and other related area. With the rapid development of computer technologies, now we can model the complex 3D architecture of

Guo, X., Zhao, C., Xiao, B., Lu, S. and Li, C., 2008, in IFIP International Federation for Information Processing, Volume 258; Computer and Computing Technologies in Agriculture, Vol. 1; Daoliang Li; (Boston: Springer), pp. 695-702. 
plants (Room et al., 1996; Reffye et al., 1997). For most crops, the aboveground morphological characteristics of them are mainly decided by upward growing stalk and leaves, which grow in different angles and orientations. Therefore the modeling of leaf is always one of the important research focuses, and many methods have been reported. Espana et al. (1999) modeled maize leaf with a rectangular 2D parameterized plane which was similar to the leaf blade. Deng XY et al. (2004) proposed a method to model static leaf based on cardinal spline and triangulation modeling. Liu XD et al. $(2002,2004)$ used Bezier curve and NURBS surface to model plant leaves. Zhao CJ et al. (2004) introduced a characterized method for simulating maize leaf based on its shape features. All the leaf models mentioned above were made purely from geometric point of view, without the concerns of visualization computing. In fact, a large number of computing are involved in the analysis of crop morphology and architecture, such as computing the light distribution in canopy (Ross, 1981; Chelle et al., 1998, 1999, Wang et al. 2004), this makes the speed of computing become a bottleneck in simulating and visualizing. Because of this, it is very necessary to refine the geometry models of crop leaves. Based on the fact that most of the shapes and surfaces of crop organs are irregular, while Non-Uniform Rational B-Splines (NURBS) is efficient for the representation of crop organs' geometry (Zhu, 2003), and it has the properties of Rational B-Splines and Non-Uniform Splines. In this article, we take the maize leaf as an example, using the point data cloud obtained by 3D digitizer, proposed a method for modeling maize leaf. This model is reconstructed by using NURBS interpolation and multi-line segment splitting algorithm; and the output mesh was utilized in the visualization and calculation of light distribution of canopy.

\section{MATIERIALS AND METHOD}

\subsection{Modeling maize leaf}

The shape of maize leaf has some obvious features: it grows on the main stalk, with a long, thin and stiff midrib supports it upward, and the edge is undulate. Using the method described by Zheng et al. (Zheng et al., 2004), obtain a group of characteristic points (data points) through digitizer, representing as $\mathrm{Q}_{\mathrm{i}, \mathrm{j}}(\mathrm{i}=0,1, \ldots, \mathrm{m} ; \mathrm{j}=0,1, \ldots, \mathrm{n})$. In order to use these points to generate NURBS surface, we define the orientation of leaf midrib as $u$ direction, and the vertical orientation to that is $\mathrm{v}$ direction, just as shown in Fig. 1 (a). Then from these data points, we can calculate the knot vectors, and 
the basis function. After that the control points of the NURBS surface can be calculated, as shown in Fig. 1 (b). Equation (1) defines arbitrary points on NURBS surface:

$$
P(u, v)=\frac{\sum_{i=0}^{n} \sum_{j=0}^{m} B_{i, k}(u) \cdot B_{j, h}(v) \cdot W_{i, j} \cdot V_{i, j}}{\sum_{i=0}^{n} \sum_{j=0}^{m} B_{i, k}(u) \cdot B_{j, h}(v) \cdot W_{i, j}},
$$

where $V_{i, j}$ is the control points, $W_{i, j}$ is the weight factor, while $B_{i, k}(u)$ and $\mathrm{B}_{\mathrm{j}, \mathrm{h}}(\mathrm{v})$ is $\mathrm{k}$ order $\mathrm{B}$-spline basis function of $\mathrm{u}$ direction and $\mathrm{h}$ order $\mathrm{B}$-spline basis function of $\mathrm{v}$ direction respectively.

Using the solved control points and knot vectors, the leaf surface model can be established, and then the NURBS surface can be generated. Fig. 1 (c) shows the wireframe of a maize leaf blade mesh generated by this method, in which the step length of interpolation is equal to one fifth of the length between two knots. That is to say, four points are interpolated between each pair of data points. Fig. 1 (d) demonstrates the rendering model with relatively more realistic effect.

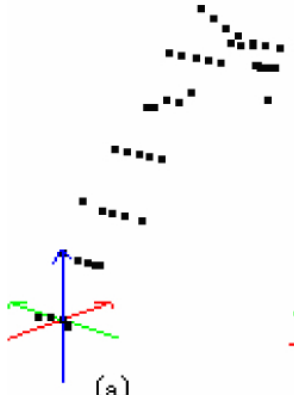

(a)

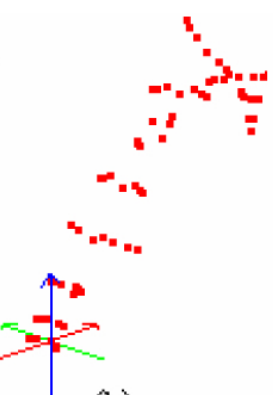

(b)

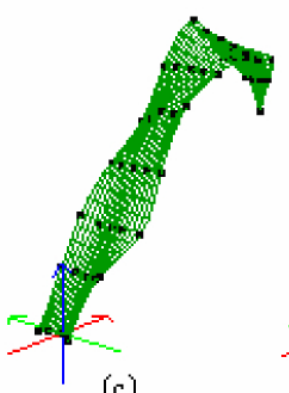

(c)

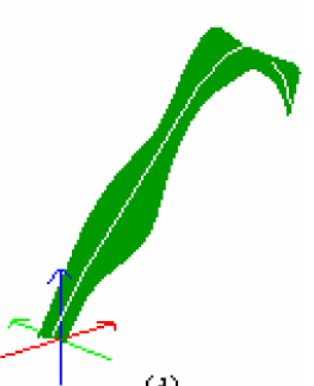

(d)

Fig. 1. The maize leaf model using NURBS interpolation

\subsection{Refining the mesh}

The surface displaying on computer screen consists of a number of mesh grids. In general, the more exquisite the surface is divided, the better rendering effect the surface has. But the smaller the grid is, the more grids are needed to generated, and this may lead to low efficiency in computing. To deal with this problem, this paper proposes a method to refine the mesh of crop leaves. User can refine the mesh of leaves according to their actual needs, and can balance the rendering effect and computing efficiency easily.

We define the mesh generated by adjacent data points as standard mesh, while the mesh generated by interpolation as reference mesh (shown in Fig. 1 (c)). When the users need to refine the reference mesh, they can reduce the number of interpolation points by increasing the step span of interpolation. 
The minimum number of interpolation points can be zero, which means the reference mesh is refined to the standard mesh. On the other hand, the standard mesh can be refined with an inverse operation of multi-line segment splitting algorithm, which can be described as follows: to a group of points, such as shown in Fig. 2, (1) select the start point and end point, and connect these two points into a line, then select the first-class characteristic point, which is the point that has the biggest distance to the line, as shown in Fig. 2 (a); (2) replace the initial line with the line connecting the characteristic point to the uplevel characteristic point, then select the second-class characteristic point; (3) continue the process until all the points are selected as characteristic point.

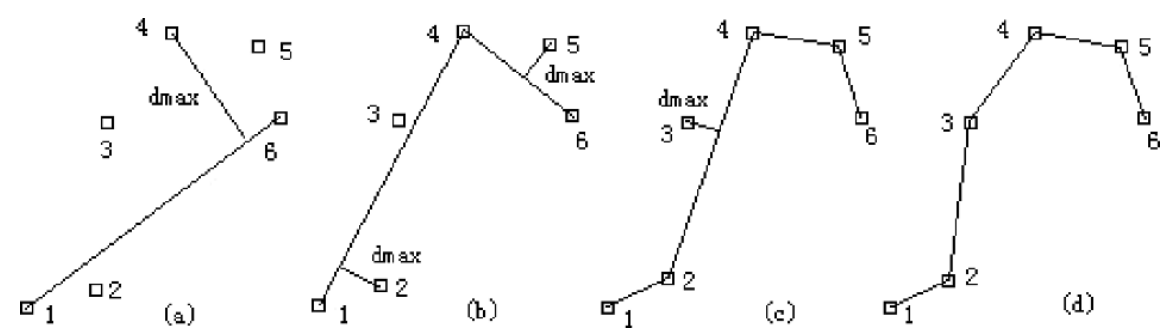

Fig. 2. Multi-line segment splitting algorithms

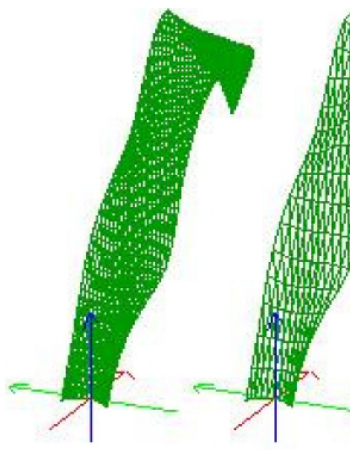

(a)

(b)

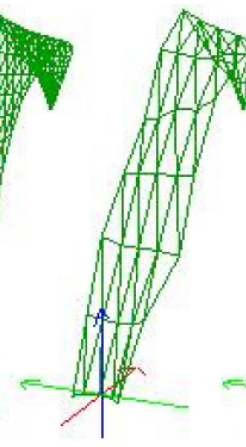

(c)

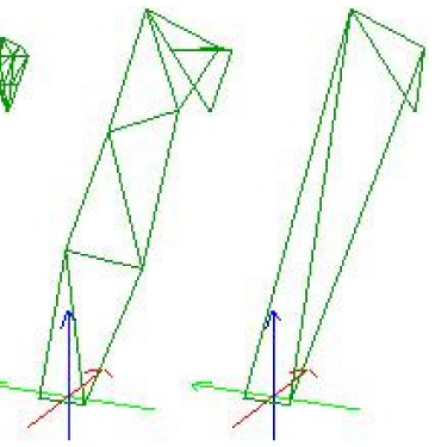

(d)

(e)

Fig. 3. Refined mesh shape of maize leaf
(a) Mesh with 1800 triangles (the reference mesh)
(b) Mesh with 440 triangles
(c) Mesh with 72 triangles (the standard mesh)
(d) Mesh with 8 triangles
(e) Mesh with 3 triangles 


\section{RESULTS}

\subsection{Maize leaf area and projection area}

Table 1. Maize leaf area and projection area of different mesh scales

\begin{tabular}{lccccc}
\hline Triangle number & 1800 & 440 & 72 & 8 & 3 \\
\hline Leaf area $\left(\mathrm{m}^{2}\right)$ & 0.123 & 0.123 & 0.122 & 0.118 & 0.102 \\
Leaf projection area $\left(\mathrm{m}^{2}\right)$ & 0.808 & 0.807 & 0.801 & 0.785 & 0.689 \\
\hline
\end{tabular}

We use the simplified geometric model of maize leaf to calculate the characteristic values, which reflect the main characteristics of leaf morphological structure, and the result was listed in Table 1. It can be found from Fig. 3 and Table 1 that the simplified scheme we proposed can significantly reduce the number of triangles of leaf model. When the number of triangles reduced from 1800 to 8 , both the inaccuracy of leaf area and leaf projection area are no more than 5 percent, but the inaccuracy increase evidently when the number of triangles is lower than 8 . Therefore our method is suitable for modeling the morphological structure, visualization calculation and analysis of maize leaf; it can dynamically meet the needs of visualization effect and computation efficiency.

\subsection{Visualization and calculation of light distribution canopy}

Using the above method, we reconstructed the canopy of maize and compared the results of a series of refined meshes.

The data used in our experiment was obtained in 2005 with a 3D digitizer 3 SpaceFastrak $(0.08 \mathrm{~cm}$ precision $)$, manufactured by an America-based company Polhemus. The canopy we calculated consists of nine maize plants arranging as 3 rows and 3 columns in the field. The row spacing is $60 \mathrm{~cm}$, and the column width is $30 \mathrm{~cm}$. The height of plant is $245 \mathrm{~cm}$. We expanded the dimension by multiplying the measured canopy up to 81 maize plants (Wang et al., 2005), which were arranged in 9 rows and 9 columns. Then the $3 \mathrm{D}$ canopy is reconstructed with series types of leaves, whose mesh consists of 440, 72, 36 and 4 triangles respectively, and the shape characteristic parameters in different refined mesh are also calculated. Fig. 4 and Table 2 show that more realistic effect can be achieved with the increase number of triangles in the leaf mesh, and more detailed description to the canopy. Meanwhile, leaf area tends to increase. 

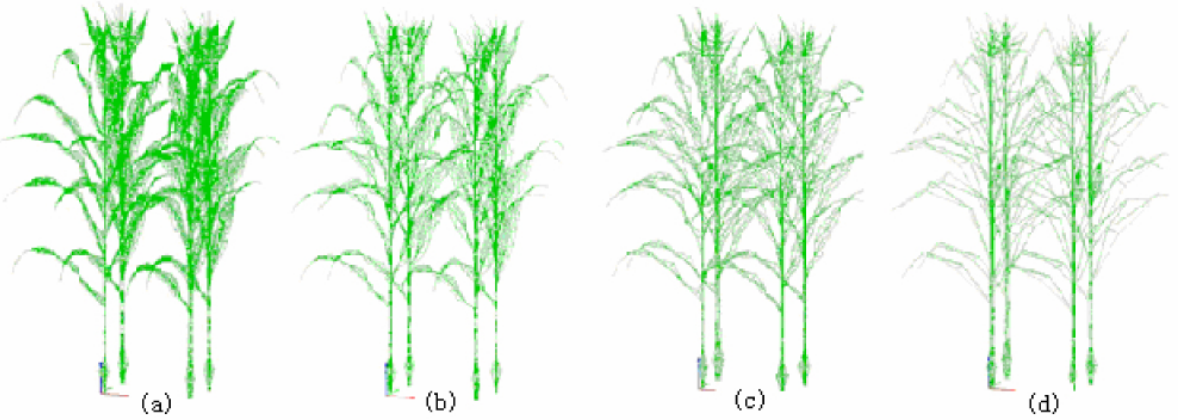

Fig. 4. The maize canopy with different mesh scales

(a) Mesh with 440 triangles

(b) Mesh with 72 triangles

(c) Mesh with 36 triangles (the standard mesh)

(d) Mesh with 4 triangle

Table 2. Maize canopy morphological character parameters of different mesh scales

\begin{tabular}{lcccc}
\hline \multirow{2}{*}{ Character parameter } & \multicolumn{4}{c}{ Mesh number } \\
\cline { 2 - 5 } & 440 & 72 & 36 & 4 \\
\hline Leaf area $\left(\mathrm{m}^{2}\right)$ & 76.5 & 75.42 & 74.79 & 60.53 \\
Total leaf projection area $\left(\mathrm{m}^{2}\right)$ & 31.6 & 30.9 & 31.76 & 24.32 \\
Vertical projection leaf area $\left(\mathrm{m}^{2}\right)$ & 10.89 & 10.7 & 10.82 & 8.94 \\
\hline
\end{tabular}

Table 3. Rate of transmitting direct light within rectangular area at different canopy heights calculated with different refined mesh

\begin{tabular}{lllll}
\hline \multirow{2}{*}{ Height $(\mathrm{cm})$} & \multicolumn{4}{c}{ Number of triangles } \\
\cline { 2 - 5 } & 440 & 72 & 36 & 4 \\
\hline 0 & 0.059 & 0.058 & 0.064 & 0.16 \\
50 & 0.13 & 0.13 & 0.12 & 0.22 \\
100 & 0.23 & 0.24 & 0.24 & 0.28 \\
150 & 0.36 & 0.38 & 0.39 & 0.52 \\
200 & 0.68 & 0.71 & 0.74 & 0.83 \\
225 & 0.87 & 0.9 & 0.91 & 0.96 \\
\hline
\end{tabular}

The radiation the crop received in nature is mainly direct radiation light from sun and diffuse light in sky. Our work implements the calculating of maize canopy direct radiation based on the mesh generated by the method described in (Mariscal et al., 2004; Wang et al., 2005). The simulating of direct radiation light can be performed as follows: simulate the sun light using a cluster of rays with an angle; the density can be determined by real requirement of light intensity. For each ray, determine whether it collides with the triangles on the mesh; and then sort the triangles collided with the 
ray according to their depth value. The triangle which has the minimum depth (with maximum z-coordinate) is radiated by the sun directly; triangles with secondary minimum depth receive second-class radiation from the sun, and we assume all others triangles do not receive any light from the sun. Above algorithm used the finite element calculation, the result meets closely to real observation. But there's too much computation in this method, and the calculation time is affected by the number of mesh grids. But using the approach introduced in this article, which uses the controllable mesh generation algorithm, the number of mesh grids can be simplified easily, and with the simplified mesh the calculation efficiency could be upgraded.

Take the light intensity calculation of the canopy at $12 \mathrm{AM}$ at the stage of maize spin silk period for example; table 3 shows different rate of transmitting direct light within rectangular area at different canopy heights of different refined mesh. The selected area is a $180 \mathrm{~cm} \times 90 \mathrm{~cm}$ plane residing inner of the canopy. We can see from Table 3 that the variation of calculation inaccuracy is not evident when the number of triangles in each leaf surface decreasing from 440 to 36 , but the computation is decreased notably. More exactly, the computing time decreases to 10 percent, this means a lot to the efficiency improvement of calculating light distribution. Users can improve the calculation by using the method of refining leaf model.

\section{DISCUSSION}

We find that when calculating the characteristic parameters and light distribution of maize canopy, the leaf area, total projection area and vertical projection area do not change evidently if the number of triangles of the leaf surface mesh has been simplified from 440 to 36, and variation of the light transmission within rectangular area at different canopy heights are lower than 10 percent, while the computation time decrease to 10 percent below. When the number of triangles is less than 36 , the leaf area, total projection area and vertical projection area decrease, and the variation increase gradually. We can conclude that the refining scheme our proposed can improve the computation evidently when the number of triangles of the leaf surface is no more than 36, and this improvement is obtained without the cost of increasing evidently the calculation variation. Our method provides a balance between the rendering effects and computing efficiency in crop leaves morphological model. 


\section{ACKNOWLEDGEMENTS}

This study has been funded by National High Tech R\&D Program of China (2007AA10Z224), Key Technologies R\&D Program of China (2006BAD10A01), Beijing Natural Science Foundation (4062015).

\section{REFERENCES}

Chelle M, Andrieu B, Radiative models for architectural modeling, Agronomie, 19, 1999, 225-240

Chelle M, Andrieu B, The nested radiosity model for the distribution of light within plant canopies, Ecological Modelling 111, 1998, 75-91

De Reffye P, Houllier F, Modelling plant growth and architecture:some recent advances and applications to agronomy and forestry, Current Science, 73, 1997, 984-992

Deng XY, Zhou SQ, Guo XY, A Static Leaf Model Based on Cardinal Spline and Triangle Faces, Computer Engineering and Applications 25, 2004, 199-201

Espana ML, Baret F, Aries F et al., Modeling maize canopy 3D architecture application to reflectance simulation, Ecological Modeling, 122, 1999, 25-43

Liu XD, Cao YF, Liu GR, The Modeling of Rice Leaf based on NUBRS, Microelectronics \& Compute, 21, 2004, 117-119

Liu XD, Jiang LH, Zhao JJ et al., The Modeling and Display of Plants Based on the Bezier Curve, Computer Engineering and Applications, 13, 2002, 97-98

Mariscal MJ, Martens SN, Light-transmission profiles in an old-growth forest canopy: Simulations of photosynthetically active radiation by using spatially explicit radiative transfer models, Ecosystems, 7, 2004, 454-467

Room PM, Hanan JS, Prusinkiewicz P, Virtual plants: new perspectives for ecologists, pathologists and agricultural scientists, Trends in Plant Science, 1, 1996, 33-38

Ross J, The Radiation regime \& architecture of plant stands. Dr. W Junk Publishers. The Hague, The Netherlands. 1981

Wang XP, Li BG, Guo Y et al., Measurement and Analysis of the 3D Spatial Distribution of Photosynthetically Active Radiation in Maize Canopy, ACTA AGRONMICA SINICA, 30, 2004, 568-576

Wang XP, Guo Y, Li BG et al., Modelling the three dimensional distribution of direct solar radiation in maize canopy, ACTA ECOLOGICA SINICA, 25, 2005, 7-12

Zhao CJ, Zheng WG, Guo XY, The Computer Simulation of Maize Leaf, Journal of Biomathemat, 19, 2005, 395-399

Zheng WG, Guo XY, Zhao CJ, Geometry Modeling of Maize Canopy, Transactions of the Chinese Society of Agricultural Engineering, 20, 2004, 152-154

Zhu XX, Modeling Technology on Free-Form Surface, Beijing: Science Press, 2003 